reservoir. The doses used led to apparently adequate levels in the CSF (Figure). Complications included a staphylococcal infection related to the surgical insertion of the Ommaya reservior and five weeks later a neutropenia, which recovered on temporarily stopping the treatment with flucytosine. Severe potassium depletion from the renal effect of amphotericin required constant monitoring and correction. The reservoir was removed after 18 doses of intrathecal amphotericin because of persistent superinfection, but it was reinstated one month later because cryptococci remained visible in, though could not be grown from, the CSF. A further 17 doses of intrathecal amphotericin were given (with intrathecal hydrocortisone to minimise the risk of chemical ventriculitis). The herpes infection on the nose responded to acyclovir. After five months in hospital he was discharged, having gained $5 \mathrm{~kg}$ in weight. Three months later his CSF still contained occasional non-viable cryptococci: further treatment with high dose oral ketoconazole and flucytosine was administered, and the Ommaya reservoir was removed.

At 17 months learning difficulties were noted, as well as persistent asymptomatic thrombocytopenia. The cerebral atrophy was unchanged on a computed tomogram of the brain. Over the next six weeks he developed visual disturbance associated with optic atrophy and spastic paraparesis. Cryptococci were not seen in the CSF, although cryptococcal antigen, initially present to a titre of 5 , remained just detectable in neat CSF.

\section{Discussion}

The clinical features in this child, including fungal, protozoal, and viral infections, weight loss, and thrombocytopenia, are consistent with AIDS. Furthermore, there is convincing laboratory evidence of retroviral infection, though this was initially unclear because the antibody response to HTLV III was atypical and undetected by our standard radioimmunoassay. ${ }^{3}$ The neonatal blood transfusions were a potential source of retroviral infection, raising the possibility that there may be a latency of many years before immunodeficiency becomes overt: unfortunately, the donors could not be traced as the blood bank concerned had since been closed. This case shows that even in an immunocompromised patient large doses of antifungal agents, together with careful attention to the inevitable complications of such treatment, can eventually eradicate cryptococcal infection. Our patient, however, has progressive neurological deterioration: this could be due to a residual effect of the cryptococcosis or its treatment, but cerebral atrophy and myelopathy are now recognised features of HTLV III infection. ${ }^{6}$

We are grateful to Dr H B Valman for his expert clinical guidance and to Dr R Wall, Department of Microbiology, Northwick Park Hospital, and Dr R Hay, London School of Hygiene and Tropical Medicine, for helpful advice and assay of antifungal drug concentrations.

\section{References}

1 Perfect JR, Durack DT, Gallis HA. Cryptococcemia. Medicine 1983:62:98-109.

2 Malkovsky M, Asherson GL, Stockinger B, Watkins MC. Nonspecific inhibitor released by $\mathrm{T}$ acceptor cells reduces the production of interleukin-2. Nature 1982;300:652-5.

${ }^{3}$ Cheingsong-Popov R, Weiss RA, Dalgleish A, et al. Prevalence of antibody to human T-lymphotropic virus type III in AIDS and AIDS-risk patients in Britain. Lancet 1984;ii:477-80.

4 Allan JS, Coligan JE, Barin F. et al. Major glycoprotein antigens that induce antibodies in AIDS patients are encoded by HTLV-III. Science 1985;228:1091-4.

5 Poiesz BJ, Ruscetti FW, Gazdar AF, Bunn PA, Minna JD, Gallo RC. Detection and isolation of type $C$ retrovirus particles from fresh and cultured lymphocytes of a patient with cutaneous T-cell lymphoma. Proc Natl Acad Sci 1980;77:7415-9.

6 Petito CK, Navia BA. Cho E-S, Jordan BD, George DC, Price $\mathrm{RW}$. Vacuolar myelopathy pathologically resembling subacute combined degeneration in patients with the acquired immune deficiency syndrome. $N$ Engl J Med 1985;312:874-9.

Correspondence to $\operatorname{Dr}$ M J Pippard, Section of Haematology, Northwick Park Hospital and Clinical Research Centre, Watford Road, Harrow, Middlesex HA1 3UJ.

Received 25 November 1985

\title{
Monitoring spontaneous respiration in the ventilated neonate
}

\author{
M SOUTH AND C J MORLEY \\ Department of Paediatrics, University of Cambridge, Addenbrooke's Hospital, Cambridge
}

SUMMARY A technique for monitoring the ventilated neonate's own respiratory efforts using a pneumatic capsule apnoea alarm is described.
Most babies, unless very sick or paralysed, continue to breathe while being ventilated, exhibiting their own respiratory pattern and certain respiratory reflexes. ${ }^{1}$ The neonate's own respiratory efforts often reduce the efficacy of mechanical ventilation 

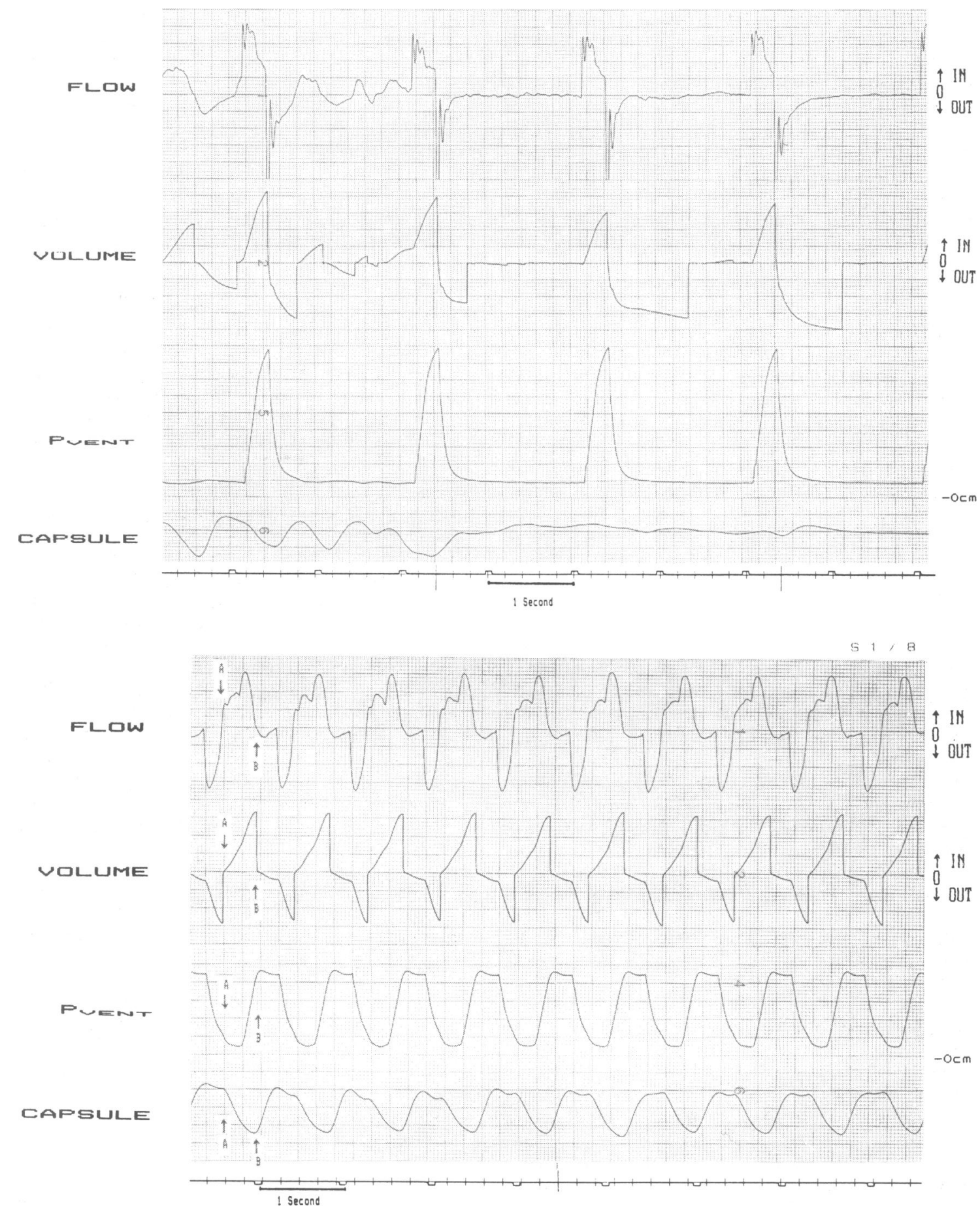

Figs. 1 (top) and 2 (bottom) Monitoring of respiratory cycles in a baby receiving mechanical ventilation. For explanation see text. 
and increase the risk of pneumothorax. ${ }^{2}$ That they may make an appreciable contribution to ventilation is shown by a need for increased mechanical ventilation after therapeutic paralysis.

If the consequences of spontaneous respiratory efforts are to be studied then a technique for monitoring them is required. Existing monitoring techniques all have disadvantages. We have previously used oesophageal pressure measurement, but making the balloons is time consuming, and passing one often disturbs the baby. The signal is disturbed by oesophageal peristalsis, and it is not suitable for long term monitoring.

\section{Equipment}

The 'MR10-Respiration Monitor' (Graseby Dynamics Ltd) uses a pneumatic capsule taped to the baby's abdomen. Respiratory movement causes capsule compression between the adhesive tape and the skin of the abdominal wall. Breaths are sensed by a silvered membrane capacitance transducer that is sensitive to changes in capsule volume. ${ }^{34}$ The amplitude of the output from the monitor unit is proportional to the change in capsule volume, and the signal can be recorded on paper or on an oscilloscope.

Random placement of the capsule on the abdomen produces a signal that is a mixture of the baby's own respiratory movements and the abdominal excursion produced by ventilator inflation.

Placement in the subxiphisternal position, with tape running across the capsule from the abdominal wall above the umbilicus to the skin overlying the xiphisternum, produces a more useful signal. In this position, ventilator inflation causes almost no compression of the capsule as there is little change in upper abdominal contour. Spontaneous inspiration involves diaphragmatic contraction with xiphisternal recession and abdominal expansion. This results in a pronounced change in upper abdominal contour, with traction on the adhesive tape causing capsule compression.

The signal produced represents spontaneous breaths, with little interference from ventilator breaths. Xiphisternal recession differentiates between them. The signal is a-c coupled, and so recordings are self centring. There is no drift, and changes in the baby's position do not alter the output, even if the baby is lying on top of the capsule.

\section{Results}

The technique has been tried over 50 times in at least 30 babies, and in each case a signal that showed spontaneous respiration without interference from the ventilator was obtained from the capsule. Gas flow was measured by a pneumotachograph in series with the endotracheal tube, as a timing reference, and in three babies it was compared with oesophageal pressure measurement. There was good timing agreement between the techniques. The measured time delay of the capsule system is between 4 and 8 milliseconds, mostly pneumatic.

In Figure 1 the capsule trace shows the lack of interference from the ventilator in a baby ventilated for recurrent apnoea. The top trace (flow) shows gas flow into (above centre zero line) and out of (below centre zero line) the endotracheal tube measured by a pneumotachograph in series with the endotracheal tube. The second trace (volume) shows the flow signal electronically integrated to give tidal volume (volume) (this integrator resets to the centre zero line at zero flow). The third trace (P vent) shows the pressure in the ventilator circuit, and the fourth trace (capsule) the signal from the pneumatic capsule. Downward deflection represents inspiration and upward deflection expiration.

On the left of the record the baby is breathing while being ventilated. The spontaneous breaths are clearly visible in the capsule trace, as well as in the flow and volume signals. On the right of the record the baby is apnoeic, and the capsule trace is almost flat. The capsule records spontaneous breaths but is not appreciably altered by ventilator inflation. Similar flat traces have been recorded from babies who have been paralysed during ventilation.

Figure 2 shows a baby breathing during mechanical ventilation, who, on clinical observation, and on first glance at this trace, would seem to be breathing regularly and in synchrony with the ventilator. More careful analysis shows that the baby is partially out of phase with the ventilator. This can be seen first by comparing the ventilator pressure trace with that of the capsule; the baby's own inspiration starts (arrow A) during ventilator expiration. The baby's own expiration starts (arrow B) midway through ventilator inflation and actually causes gas flow up, rather than down, the endotracheal tube. Careful analysis of the 'double humped' appearance of inspiratory and expiratory flow traces also leads to this conclusion. Comparing gas flow or tidal volume signals with that from the capsule confirms the timing accuracy of the capsule technique.

\section{Discussion}

The technique provides a signal that represents the baby's own respiratory pattern with little or no interference from the ventilator. The system is simple and non-invasive. It can be used continuously 
and does not interfere with any aspect of the baby's care.

The amplitude of the signal is a qualitative reflection of change in intrathoracic pressure and is not a measure of tidal volume. Quantitative timing measurements can be made and phase relations between spontaneous respiration and ventilation studied. The capsule can be used to detect the baby breathing out of phase with the ventilator, even when clinical observation would not suggest this. This pattern of interaction can reduce the efficacy of ventilation and increases the risk of pneumothorax. ${ }^{2}$

This method should prove to be a useful tool in ventilation research and in neonatal clinical practice.

We thank Professor J A Davis. Dr N R C Roberton, and Dr G Gandy for their encouragement and advice. Sr J Pool for making the oesophageal balloons, and the nursing staff of the neonatal unit at the Rosie Maternity Hospital. This work is supported by the University of Cambridge Baby Research Fund.

\section{References}

1 Greenough A, Morley CJ, Davis JA. Respiratory reflexes in ventilated premature babies. Early Hum Dev 1983;8:65-75.

2 Greenough A, Morley CJ, Wood S, Davis JA. Pancuronium prevents pneumothorax in ventilated premature babies who actively expire against positive pressure inflation. Lancet $1984: i: 1-3$.

${ }^{3}$ Wright BM. An abdominal respiration detector. J Physiol (Lond) 1977;271:11-2.

4 Wright BM, Callan K, eds. Proceedings of the 3rd international symposium on ambulatory monitoring. London: Academic Press, 1980:329-34.

Correspondence to $\operatorname{Dr} M$ South, Department of Paediatrics, University of Cambridge, Level E8, Addenbrooke's Hospital, Cambridge CB2 2QQ.

Received 26 November 1985

\title{
Home care team in accident and emergency
}

\author{
E GLUCKSMAN, S S TACHAKRA, S PIGGOTT, AND H LEA
}

Central Middlesex Hospital, London

SUMMARY We describe experience with a community paediatric service designed to provide more convenient follow up for children and their families and to reduce the number of children returning unnecessarily to the accident and emergency department.

Every year one in six children are likely to attend accident and emergency departments, making up roughly $20 \%$ of the new attendances to departments in England and Wales. ${ }^{1}$ It has been suggested repeatedly that accident and emergency departents may not be the most satisfactory places for seeing and treating sick children. ${ }^{2}$ Nevertheless, many families use the accident and emergency department, and commonly as an alternative to the general practitioner's surgery. ${ }^{3}$ Although it may be difficult to alter substantially the pattern of new attendances by children, it may be practical to identify children who need follow up after the initial visit but who need not return to accident and emergency departments.

\section{Patients and methods}

In 1981 the role of an existing paediatric home care team was extended to follow up selected children attending the accident and emergency department. Children who might not require hospital facilities for follow up included those with: medical problems for example, vomiting or diarrhoea; simple sutured lacerations; minor burns; and other soft tissue injuries where dressing changes were required - for example, dog bites. Children under 12 who lived in the Brent Health District were eligible for referral and the service was available seven days a week and provided by three paediatric sisters, all with SRN RSCN qualifications. Medical responsibility remained with the accident and emergency staff, and if problems arose nurses referred children to the department.

If the child's injury or illness was suitable for referral the accompanying parent or guardian was given the choice of a community visit or reattendance to the accident and emergency department. As an indication of the usefulness of the service a brief questionnaire was given to families in which an adult understood English. Visits and treatments were carried out at school, nursery, childminder, or home, whichever was most suitable. Parents were given a card advising them to call if worried.

\section{Results}

Between 1 December 1982 and 30 November 1983 there were a total of 36971 new patient attendances 